All this smacks of transvection and other homology-based silencing phenomena, which are increasingly recognized as important developmental mechanisms ${ }^{14}$. But the mark on the paternal X chromosome might not be indelible, because male mice who inherit their X chromosome from their fathers are not apparently developmentally impaired ${ }^{15}$. Notably, Bean et $a l .{ }^{2}$ found that the imprint of the C. elegans paternal X chromosome disappears after several rounds of mitosis as it accumulates histone modifications. Although the observed histone mark disappeared, we don't yet know if the underlying imprint also disappeared.

Now the questions begin: how is lack of pairing recognized, how is the imprint established and what is the underlying imprint? One thing is clear: the special status of the unpaired X chromosome of XO males is identified through the histone code. Although many will assume that the special X chromosome is transcriptionally silent, this has not yet been rigorously demonstrated. But whatever the epigenetic mark and whatever its function, knowledge of the roles of modified histones will take us closer to discovery.

This paper provides grist for the experimental mill of many of us: those interested in meiosis, reproduction, embryogenesis, transcriptional control, chromatin and evolution. It has wide implications to be tested in several systems, and I, for one, am quite eager to see where this takes us!

1. Reik, W. \& Walter, J. Nat. Rev. Genet. 2, 21-32 (2001).

2. Bean, C.J., Schaner, C.E. \& Kelly, W.G. Nat. Genet. 36, 100-105 (2004).
3. Kelly, W.G. et al. Development 129, 479-492 (2002).

4. Reuben, M. \& Lin, R. Dev. Biol. 245, 71-82 (2002).

5. Jenuwein, T. \& Allis, C.D. Science 293, 1074-1080 (2001).

6. McKee, B.D. \& Handel, M.A. Chromosoma 102, 71-80 (1993).

7. Peters, A.H.F.M. et al. Cell 107, 323-337 (2001).

8. Shui, P.K. \& Metzenberg, R.L. Genetics 161, 1483-1495 (2002)

9. Lee, D.W., Pratt, R.J., McLaughlin, M. \& Aramayo, R. Genetics 164, 821-828 (2003).

10. Reinke, V. et al. Mol. Cell 6, 605-616 (2000).

11. Wang, P.J., McCarrey, J.R., Yang, F. \& Page, D.C. Nat. Genet. 27, 422-426 (2001).

12. Moss, S.B., VanScoy, H. \& Gerton, G.L. Mamm. Genome. 8, 37-38 (1997).

13. Huynh, K.D. \& Lee, J.T. Nature advance online publication, 7 December 2003 (doi:10.1038/nature 02222).

14. Wu, C. \& Morris, J.R. Curr. Opin. Genet. Dev. 9 , 237-246 (1999).

15. Handel, M.A. \& Hunt, P.A. Mol. Reprod. Dev. 28, 337-340 (1991).

\title{
Diverse powerhouses
}

\section{David R Thorburn}

\begin{abstract}
Mitochondria in different tissues vary in number, morphology, ultrastructure, respiratory capacity and involvement in specific metabolic pathways. A comparison of the proteome of mitochondria from different tissues has identified the extent of the underlying variation in protein composition and how this may be determined by tissue-specific networks of coregulated genes.
\end{abstract}

For an organelle that once seemed to be loved only by hard core biophysicists, the mitochondrion has come a long way. Severe disorders of mitochondrial oxidative phosphorylation (OXPHOS) are now recognized as the most common group of inborn errors of metabolism, affecting at least 1 in 5,000 individuals ${ }^{1}$. Mitochondria are best known as the cell's energy source as producers of ATP. But, they also have pivotal roles in generating reactive oxygen species, calcium metabolism and cell death. Thus, it is not surprising that mitochondrial dysfunction contributes to diverse pathologies including neurodegeneration, diabetic complications and tumorigenesis. A recent study by Vamsi Mootha and colleagues ${ }^{2}$ in Cell provides a basis for connecting mitochondrial pathologies with molecular etiology by identifying new mitochondrial proteins and profiling the extent of their tissue-specific diversity.

David R Thorburn is at The Murdoch Childrens Research Institute and Genetic Health Services Victoria, Royal Children's Hospital, and Department of Paediatrics, University of Melbourne, Melbourne, Australia.

e-mail:david.thorburn@mcri.edu.au

\section{Compiling the list}

Over the past few years, different strategies have been used in attempting to determine the total number of proteins in mitochondria. These include epitope-tagging, systematic functional screening of whole-genome

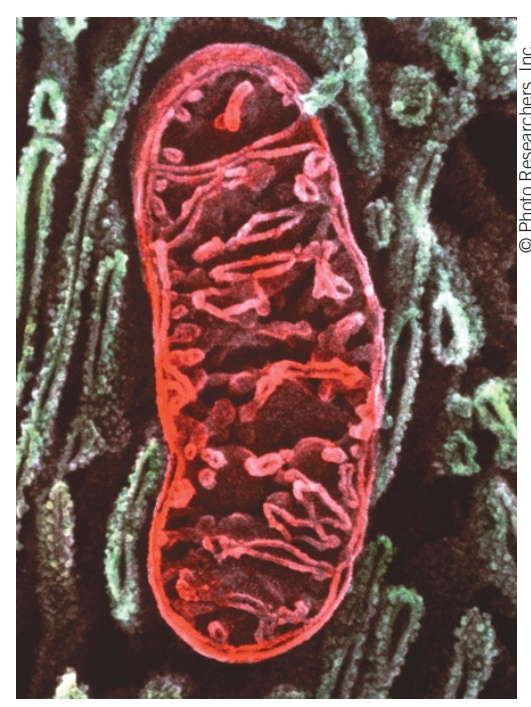

Figure 1 Colored high resolution scanning electron micrograph of a single mitochondrion in the cytoplasm of an intestinal epithelial cell. pools of mutants and proteomic analyses of highly purified mitochondria. Bioinformatic analyses have also been used to predict proteins with a classical mitochondrial targeting sequence or genes that are coregulated with genes encoding known mitochondrial proteins. These complementary approaches are necessary to overcome the various limitations in sensitivity and specificity of each method. Recent studies using such approaches in yeast are consistent with an estimate of 800-1,000 different mitochondrial proteins ${ }^{3-6}$.

Yeast mitochondria are relatively simple and uniform, but mammalian mitochondria vary widely between different tissues and often don't resemble the text-book version (Fig. 1) in morphology or composition ${ }^{7}$ (Table 1). The mitochondrial proteome of mammals is probably much larger and more diverse than that of yeast, but its size and variability have not been ascertained. Computational analysis predicts up to 4,000 mitochondrial proteins in humans ${ }^{3}$, which may be a true reflection of the complexity and diversity of our mitochondria or an overestimate caused by a systematic artifact. The first large-scale proteomic analysis of mammalian mitochondria, using human heart tissue, was 
published recently and concluded that at least 615 different proteins were present ${ }^{8}$.

Mootha and colleagues now report a proteomic survey of mitochondria purified from mouse brain, heart, kidney and liver ${ }^{2}$. They identified 399 mitochondrial proteins using liquid chromatography tandem mass spectrometry. Of these proteins, 75 were new and 88 had recently been identified as mitochondrial proteins 8 . Although some could be contaminants, most seem authentic based on multiple lines of evidence. Mootha et al. compiled these proteins with known mitochondrial proteins and obtained a list of 591 distinct mitochondrial proteins, called, in Hollywood parlance, the mito-A list.

\section{Variation and cooperation}

Only $40 \%$ of the previously known mitochondrial proteins were present in all four mouse tissues, and after correcting for imperfect sensitivity and reproducibility, it was predicted that $\sim 85 \%$ of the mitochondrial proteins detected in one tissue would be detected in a different tissue. Analysis of published RNA expression levels also suggested that about half the mitochondrial genes were expressed in all four tissues. The protein and RNA data were consistent with a simple model in which half the proteins in the mitochondrial proteome are ubiquitous and half are tissue-specific, with a $50 \%$ probability of being expressed in a given tissue. The true situation is undoubtedly more complex, with the tissue-specific component likely to include some proteins expressed in perhaps a single tissue and others expressed in most but not all tissues. But this model gives a framework on which to base further investigations.

For two-thirds of the mito-A genes, previously published expression data were available for 45 mouse tissues, and a number of clusters or subnetworks of genes were identified with correlated expression. Not surprisingly, the largest cluster was enriched for genes related to OXPHOS. This group would be expected to have quantitative, rather than absolute, differences in expression and was most prominent in tissues with the highest oxidative capacity. Other clusters corresponded to tissue-specific metabolic pathways, such as steroidogenesis in adrenal cortex and heme synthesis in bone marrow. Clustering allows preliminary functional

Table 1 Examples of the extent of mitochondrial variation between tissues ${ }^{7}$

\begin{tabular}{ll}
\hline Feature & Range of variation \\
\hline Morphology & Spheroid (liver), reticulum (cultured cells) \\
Ultrastructure of cristae & Lamellar (muscle), tubular (adrenal cortex) \\
DNA copy number per cell & 50 (sperm) to 200,000 (oocyte) \\
OXPHOS capacity (cytochrome c content) & 2 (skin) to 136 (heart muscle) $\mu$ g/g wet weight \\
Specific enzyme systems & $\begin{array}{l}\text { Steroidogenesis (adrenal cortex), heme biosynthesis } \\
\text { (bone marrow) }\end{array}$ \\
\hline
\end{tabular}

annotation of proteins with unknown or unanticipated roles; for example, the OXPHOS subnetwork included 11 proteins not previously associated with OXPHOS.

Analysis of published expression data for 10,000 nuclear and mitochondrial genes identified 470 non-mito-A genes whose expression profiles were most similar to genes in the mito-A list; comprising the 'mitochondrial neighborhood'. Many of these probably encode authentic mitochondrial proteins not detected by proteomics, and others may encode nuclear transcription factors, cytosolic chaperones and other proteins and RNAs whose expression is linked to mitochondrial genes. Notably, and in keeping with the authors' previous results from a human expression data set ${ }^{9}$, the mitochondrial neighborhood contains only about half of the mito-A genes. This might be expected if there are large numbers of mitochondrial proteins expressed in a relatively unique manner (e.g., with high tissue-specificity) shared by only small numbers of other mitochondrial proteins.

\section{Deducing origins}

A popular theory for the origin of mitochondria holds that they are derived from a eubacterial symbiont. Some mitochondrial proteins are thought to have derived from genes introduced by this ancient bacterial invader to the host nuclear genome, whereas others are thought to have been recruited to the evolving mitochondrion from the host genome to allow for protein import, ATP export and other (tissue-)specific functions. The ancestral proteins in the mito-A list-those with homologs in eubacteria-tended to share their pattern of gene expression with more mitochondrial proteins than others, that is, they had larger numbers of close neighbors. Previous studies suggest that, at least in yeast, these ancestral proteins are more likely to be translated on polysomes in the immediate vicinity of mitochondria than on free cytosolic polysomes ${ }^{10}$. So, it seems that a core group of ubiquitous mitochondrial proteins may have retained a distinct mechanism of coregulated gene expression.

Combining the two recent proteomics studies results in a list of more than 700 proteins in the mammalian mitochondrial proteome $e^{2,8}$. Further studies are clearly needed to define the true number, which is probably at least twice this value, given the extent of differences between different tissues, the likelihood that many proteins have low abundance and the number of potential mitochondrial proteins predicted by gene expression analyses. Thus, it is reasonable to conclude that at least $5 \%$ of mammalian genes, and perhaps up to $10 \%$, encode proteins located in the mitochondria of one or more tissues.

The expanding spectrum of mitochondrial proteins offers a new range of candidate genes for involvement in OXPHOS disorders and risk factors for many common diseases. Analysis of coregulated mitochondrial genes, and the transcription factors regulating the networks, also offers an approach to understanding the pathogenesis of mitochondrial dysfunction in conditions such as diabetes ${ }^{11}$.

1. Skladal, D., Halliday, J. \& Thorburn, D.R. Brain 126, 1905-1912 (2003).

2. Mootha, V.K. et al. Cell 115, 629-640 (2003).

3. Richly, E., Chinnery, P.F. \& Leister, D. Trends Genet. 19, 356-362 (2003).

4. Kumar, A. et al. Genes Dev. 16, 707-719 (2002).

5. Steinmetz, L.M. et al. Nat. Genet. 31, 400-404 (2002).

6. Sickmann, A. et al. Proc. Natl. Acad. Sci. USA 100, 13207-13212 (2003).

7. Tyler, D. The mitochondrion in health and disease. 477-520 (VCH, New York, 1992).

8. Taylor, S.W. et al. Nat. Biotechnol. 21, 281-286 (2003).

9. Mootha, V.K. et al. Proc. Natl. Acad. Sci. USA 100, 605-610 (2003).

10. Marc, P. et al. EMBO Rep. 3, 159-164 (2002)

11. Mootha, V.K. et al. Nat. Genet. 34, 267-273 (2003). 